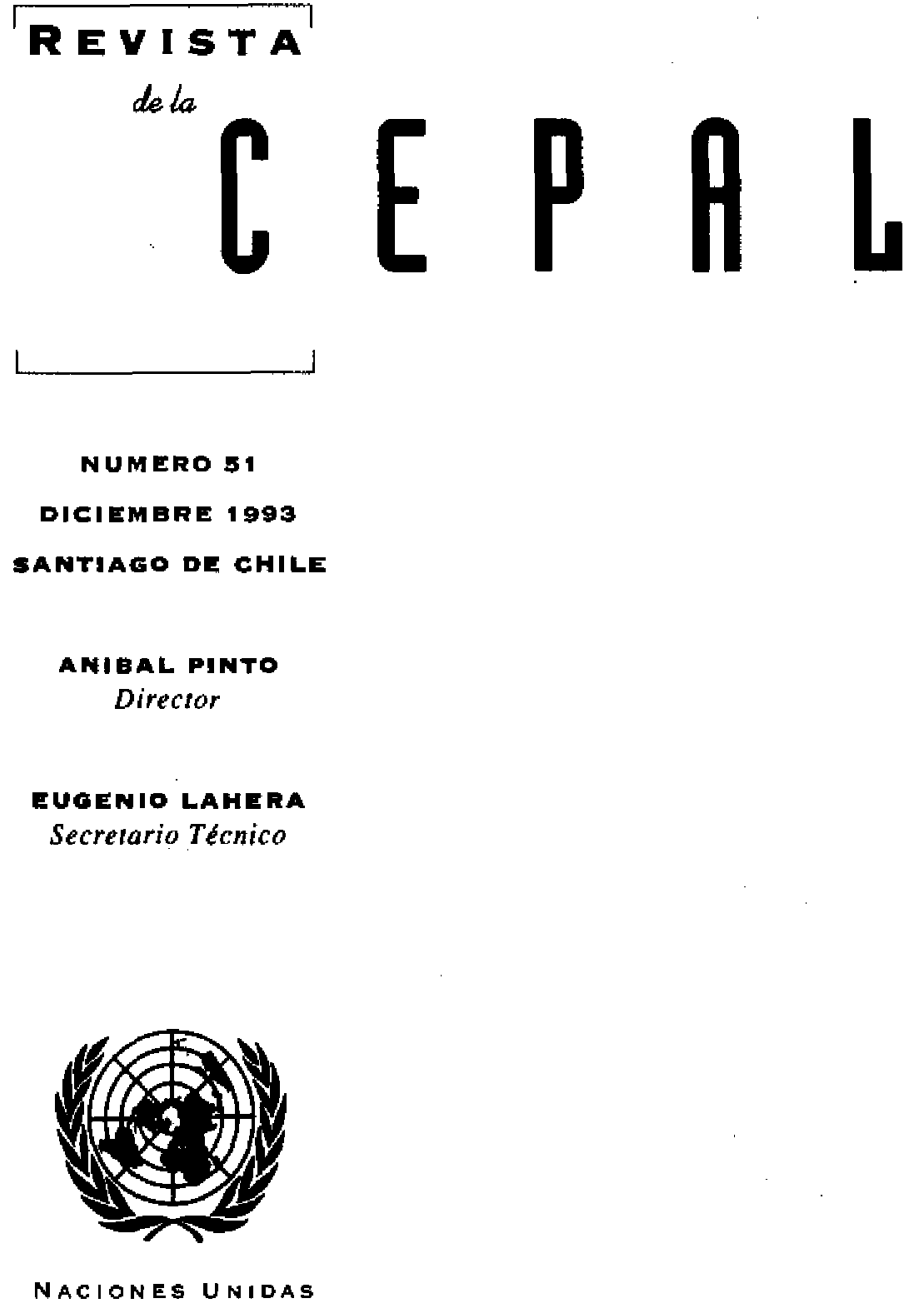


Inauguración de la Sala Fernando Fajnzylber y lanzamiento de la Revista de la CEPAL N² $\mathbf{5 0}$

Gert Rosenthal y Alejandro Foxley

Las transnacionales y la Industria en los paises en desarrollo

Michael Mortimore

¿Dónde estamos en política Industrial?

El desafío de la competitividad Industrial

Rudolf M. Buitelaar y Leonard Mertens

Integración y desintegración social rural

Martine Dirven

Los pueblos Indigenas y la modernidad

John Durston

Productividad y trabajo de la mujer en los Estados Unidos

Inés Bustillo y Nancy S. Barrett

Efectos de las corrientes de capltal sobre la base monetarla

111

Helmut Reisen

Viejas y nuevas políticas comerclales

Daniel Lederman

Integración y desviación de comerclo

Renato Baumann

Integración europea y comerclo latinoamericano

Miguel Izan

El actual debate sobre los recursos naturales

Fernando Sánchez Albavera

Orlentaclones para los colaboradores de la Revista de la CEPAL 


\section{¿Dónde estamos en política industrial?}

\section{Wilson Peres Núñez}

Asesor Técnico Principal del Proyecto CEPAL/TNUD

"Diseño de politicas para el fortalecimiento de la capacidad de innovación tecnológica y elevación de la competisividad internacional en el ambiro empresarial".
Resulta paradójico que en los años ochenta, mientras la polítjca industrial tendía a desaparecer como tema de reflexión aca démica y era relegada de la toma de decisiones en la mayoría de los países latinoamericanos, continuaba siendo la base de importantes acciones e instrumentos que se aplicaban en los países y regiones desarrollados y de reciente industriatización. Durante ese mismo período diversas insuficiencias y distorsiones gestadas en la dinámica industrial hicieron que al iniciarse el decenio de 1990 las perspectivas para ese campo de la política económica fuesen más positivas. La política industrial de este decenio será diferente a la que predominaba en períodos caracterizados por economías en gran medida cerradas, industrias no globalizadas y políticas estatales que se basaban principalmente en subsidios, en la inversión directa en empresas estatales y en la administración de la protección comercial. La sección I del artículo resalta un conjunto de hechos estilizados que muestra las principales caracteristicas de la política industrial tradicional y los límites a los que ella se vio enfrentada. La sección II analiza algunas experiencias desarrolladas desde mediados de los años ochenta, $y$ pone de relieve que el surgimiento de nuevos enfoques en América Latina, así como diversos elementos externos a la region, indica que la política industrial no puede ser excluida en ta tarea de recuperar el crecimiento e integrar los países de la región a la economía mundial. Por último, la sección III presenta conclusiones que apuntan, por un lado, a poner de relieve algunos temas permanentes en el análisis de la política industrial y, por otro, a delinear diversas propuestas sobre la manera de encarar estos temas para formular una política más eficaz y, sobre todo, más eficiente que en el pasado. 


\section{I}

\section{La política industrial tradicional}

Cualquier intento de sistematizar la amplia experiencia de los países latinoamericanos en política industrial exigiría un muy extenso análisis de casos de decisión y aplicación de políticas, lo que supera los objetivos de este artículo. Sin embargo, esa experiencia puede ser resumida, aun a riesgo de perder parte de la riqueza que la caracteriza, en un conjunto de hechos estilizados que ponen de relieve los elementos fundamentales de esa práctica, y que se presentan a continuación.

La política industrial en la región y la correspondiente selección de estrategias han tendido a reflejar las preferencias teóricas (o al menos analíticas) de las principales esferas de decisión de política, más que consideraciones pragmáticas.

$\mathrm{Si}$ bien en los comienzos de las experiencias industrializadoras de los países más grandes de la región hubo enfoques de política que sólo fueron sistematizados (o incluso considerados como tales) con posterioridad, la inconformidad con la dinámica industrial presente a finales de los años sesenta y comienzos de los setenta llevó a intentos formales de planificación. En esa dinámica influyeron de manera determinante tanto la pérdida de eficacia de los instrumentos normativos fundamentales como la cooptación de numerosas instancias decisorias por parte de grupos de interés.

La multiplicidad de organismos e intereses y el rendimiento decreciente de la política en vigor llevaron a una saturación de instrumentos que muchas veces eran contradictorios entre sí. En este marco, la formalización de las estrategias y de los instrumentos de política en planes y programas fue resultado de la voluntad de los decisores de la política de cambiar las estructuras, comportamientos y desempenos industriales prevalecientes, y se concretó en experiencias sustentadas en consideraciones analíticas más o menos amplias. En algunos casos, los planteamientos básicos respondían a visiones de cómo se debía concebir el propio proceso de crecimiento económico; mientras que en otros, estaban más vinculados a

$\square$ El autor agradece los comentarios y las sugerencias de Antonio Barros de Castro, Fábio Erber, Ricardo Ffrench-Davis, Jorge Katz, Carla Macario, Yoshiaki Nakano y Joseph Ramos. la generalización de proposiciones de equilibrio parcial sustentadas por los análisis de organización industrial.

Los marcos teóricos de la experiencia de política industrial tendieron a seguir fundamentalmente las líneas de la tradición Kaldor-Verdoorn; ésta resaltaba el papel del crecimiento del mercado como motor del incremento del empleo y de la productividad del trabajo, el que tendría lugar fundamentalmente en la industria manufacturera (Kaldor, 1966). Las ganancias de competitividad que se obtendrían a partir del crecimiento en condiciones en las que prevalecían las economías de escala permitirían no sólo ampliar los mercados internos, sino también conquistar los mercados externos. La protección comercial para avanzar en la sustitución de importaciones era entonces el mecanismo fundamental para internalizar los efectos del círculo virtuoso crecimiento-productividad-crecimiento, particularmente porque en general se dudaba de que los principales mercados internacionales tuviesen la capacidad de absorber las exportaciones manufactureras latinoamericanas. Cuando se quería reducir los efectos negativos de una protección excesiva -el sesgo antiexportador, la baja presión para incorporar progreso técnico u otros-se tendía a establecer subsidios generalizados a los costos de producción mediante, por ejemplo, importaciones de bienes de capital con arancel cero o extremadamente bajo, tasas de interés preferentes y precios de insumos producidos por empresas estatales inferiores a los costos de producción.

En las políticas sectoriales predominaban los enfoques basados explícita o implícitamente en el modelo de estructura-conducta-desempeño, desarrollado en la tradición de Mason, Scherer y Bain (Scherer y Ross, 1990). Este modelo permitía efectuar análisis con una fuerte base empírica e identificar políticas para modificar el desempeño industrial a través de cambios en la estructura productiva o en la conducta empresarial. En general, se consideraba que las modificaciones de estructura debían lograrse con la regulación o la intervención estatal directa, mientras que los cambios de conducta debian ser inducidos mediante modificaciones de las rentabilidades relativas. El modelo de estructura-conducta-desempeño 
aparecía como particularmente útil en situaciones de economía cerrada en las que la concentracion industrial y el poder del mercado estaban directamente correlacionados.

Las políticas industriales de la región tendieron a exhibit una continuidad bastante excepcional, en particular si se tiene en cuenta los frecuentes cambios de planes y programas cada pocos años, que a menudo buscaban, precisamente, mostrar que se hacía una política diferente a la de la administración anterior. Hacia comienzos de los años ochenta la planeación industrial intentaba, en la mayor parte de los casos, combinar la sustitución de importaciones y el fomento a las exportaciones, en especial de manufacturas, para reducir el desempleo y aumentar la producción de bienes de consumo esenciales. En general, se trataba de impulsar la integración de cadenas productivas, siempre con miras a fomentar la producción de insumos intermedios de uso generalizado $\mathrm{y}$, en algunos países, la de bienes de capital. Los instrumentos de política eran también básicamente similares (fomento, protección y regulación), aunque se observaba diferencias de énfasis o de concepciones entre las diferentes experiencias.

Aunque en los años setenta hubo un importante crecimiento industrial en muchos países de la región, 1 a comienzos de los años ochenta comenzó a observarse una creciente ineficacia de la planificación industrial. Esta ineficacia se habría debido fundamentalmente i) a una persistente falta de coherencia entre los niveles de planeación sectorial y macroeconómica; ii) a estrategias que no concitaban la adhesion de niveles importantes de la administración o eran combatidas o ignoradas por importantes entidades públicas que debían participar en su implementación; iii) a la deficiente implementación de los programas, que no se concretaban en partidas presupuestarias por su falta de metas a nivel de instrumentos de política, y iv) a la poca articulación con los agentes productivos privados, los que en respuesta no le brindaban confianza $\mathrm{y}$ adhesión.

La aceptación de este diagnóstico por amplios círculos decisorios llevó a una crisis de la planificación industrial que luego se extendió al conjunto de

\footnotetext{
${ }^{1}$ Naturalmente, diferentes concepciones económicas discrepan acerca de si ese crecimiento se logró debido o pese a la política industrial, en especial en un contexto de amplio financiamiento externo. Véase, por ejemplo, Chami Batista (1992) y Dias Car neiro (1990) para el caso de Brasil, y Peres (1989) para el de México.
}

la política industrial. Este fenómeno no fue exclusivo de América Latina. Así, la OCDE (1987) señala que, luego de un auge del intervencionismo estatal en sus países miembros en los años setenta y el inicio de los ochenta, la ola intervencionista perdió fuerza e incluso retrocedió a sus niveles más bajos dẹl período previo a la crisis de los años setenta. A la vez, se cuestionó la validez de algunos de los principios que habían inspirado la política industrial de los gobiernos. Este cuestionamiento, que se inició en los países industrializados, se expandió rápidamente en la región latinoamericana, tanto por la influencia de las ideas desarrolladas en esos países, como por elementos que surgían de la propia experiencia de numerosos países de la región en materia de política industrial.

La validez de la política industrial fue cuestionada en general debido a errores que habían llevado a sobreestimar los costos de las fallas del mercado y a subestimar aquéllos asociados con las fallas del gobierno. En particular, las políticas industriales habrían encontrado límites impuestos por los hechos siguientes. En primer lugar, las opciones abiertas a los gobiernos se vieron reducidas por la creciente internacionalización del comercio y la producción, y por una cada vez más difusa separación entre sectores productivos; por otra parte, el dinamismo de la tecnología y los mercados hizo cada vez más difícil y riesgoso para los gobiernos el intento de controlar las variables del contexto de la planificación. En segundo lugar, las políticas aplicadas eran difíciles de evaluar y controlar, lo que las hizo extenderse más allá de su horizonte de validez, fundamentalmente por la labor de grupos de interés. La ausencia de criterios claros para decidir el comienzo y el fin de su aplicación hizo más difícil concretar el objetivo, muchas veces manifestado, de utilizar los instrumentos normativos en un marco de estricta temporalidad; asimismo, la cooptación de órganos decisorios por grupos de interés dificultó aún más la eliminación de medidas normativas cuyo horizonte de validez ya había expirado. $\mathrm{Y}$ en tercer lugar, algunas políticas tenían una limitada eficacia intrínseca, principalmente porque su complejidad elevaba la probabilidad de conflicto con los objetivos de otras. Además, la coordinación de políticas planteó problemas a menudo insuperables, en especial por la ausencia de un vínculo claro entre política industrial y política macroeconómica.

El consenso surgido a mediados de los años ochenta sobre el tipo de reformas que necesitaba 
América Latina tendió a negar la validez e incluso la posibilidad de usar instrumentos que habían sido tradicionales en la política industrial de la región (Balassa, Bueno, Kuczynski y Simonsen, 1986). Así, por ejemplo, las políticas que tendieron a aceptarse en materia de apertura comercial, tasa de interés, orientación del gasto público, privatización, desregulación y derechos de propiedad intelectual entraron en conflicto directo con los instrumentos en que se habían sustentado las políticas industriales y los desplazaron. La percepción de que las políticas como tales habían perdido validez, unida a la imposibilidad o indeseabilidad de utilizar sus instrumentos tradicionales, las hizo desaparecer o pasar a cumplir un papel puramente declarativo de un interés genérico por "la industria", el que debía equilibrarse con el interés que merecían otros sectores económicos. Este intento de equilibrar o de no discriminar entre sectores llevó a un progresivo relegamiento de las políticas industriales de alcance sectorial, las que se basaban precisamente en el uso intensivo de los instrumentos señalados anteriormente.

\section{II}

\section{Algunos cambios de fines de los años ochenta y principio de los noventa}

En los años ochenta, muchos países de América Latina parecieron llegar a conclusiones similares a las señaladas en la sección anterior respecto a la política industrial, y ésta tendió a desaparecer del conjunto de políticas relevantes o, a lo sumo, a ser identificada con la eliminación de las estructuras y comportamientos que se asociaban a las políticas industriales tradicionales. $^{2}$

Pese a lo anterior, las prácticas de política industrial continuaron teniendo un peso importante en la mayoría de los países desarrollados de Europa (OCDE, 1987), ${ }^{3}$ en tanto que las características ampliamente estudiadas del Estado desarrollista de Japón y las economías de industrialización reciente del este de Asia mantenían sus rasgos fundamentales. ${ }^{4}$ De las políticas

\footnotetext{
${ }^{2}$ Naturalmente, la virtual desaparición de la política industrial como tal no impidio la adopción de importantes medidas en los campos de la privatización y de la refinanciación de la deuda externa (por ejemplo, autorizaciones para canjes de deuda por inversión), las que tuvieron amplio efecto sobre la dinámica de determinados sectores y empresas industriales.

${ }^{3}$ En última instancia, el consenso sobre el conjunto de políticas viábles tendió a concentrarse en tres objetivos fundamentales; i) la nivelación de las condiciones de la pugna competitiva entre empresas de distinto tamaño o provenientes de regiones con diferentes grados de desarrollo; ii) el fomento de la innovación y difusión de tecnologías debido al importante contenido de cxternalidades en estas actividades, y iii) la compensación, al menos parcial, de desincentivos a la inversión causados por políticas macroeconómicas (por ejemplo, las que dan lugar a aumentos de la tasa de interés).

${ }^{4}$ Para un interesante análisis de cómo el estilo de política industriàl típico de los países de industrialización reciente del este de Asia se ha mantenido incluso en sus actuales etapas de desarrollo
}

formuladas o propuestas en ese período puede decirse hoy, con la ventaja de la perspectiva temporal, que abrieron caminos y que pueden llegar a determinar el alcance posible de la política industrial en los años noventa. A continuación se analizan algunas de esas experiencias y propuestas.

\section{La politica posible en áreas desarrolladas: Ontario, 1988}

A partir de un trabajo iniciado un par de años antes, el Consejo del Primer Ministro de Ontario dio a conocer en 1988 un informe en el que planteaba un amplio conjunto de objetivos y de instrumentos de política para reestructurar la economía provincial en un marco de globalización, dinamismo tecnológico y creciente competencia externa (Ontario, Premier's Council, 1988).

Los problemas que enfrentaba la provincia canadiense de Ontario tenían rasgos que eran comunes tanto a países desarrollados como a aquéllos de industrialización intermedia basada en recursos naturales. Por una parte, la provincia tenía una estructura industrial y de innovación moderna comparable a la de otras regiones de elevado desarrollo (por ejemplo, Baden Wurtenberg, Lombardía o Cataluña) y contaba con empresas competitivas en el ámbito internacional (como Northern Telecom). Pero por otra, su base pro-

relativamente elevado, véase Liml (1993) para el caso de Singapur, y Choi (1993) para el caso de la República de Corea. 
ductiva adolecía de graves problemas derivados del peso que tenían en la economía provincial las actividades de elaboración primaria de recursos naturales como las industrias del papel y la celulosa, de alimentos procesados, siderúrgica y química) y las industrias sujetas a fuerte competencia (como la de partes para automoviles).

La propuesta industrial que hizo el Consejo del Primer Ministro se basaba en proposiciones analíticas que, desde su propio punto de vista, se distanciaban apreciablemente de la forma tradicional de hacer política industrial. En particular, ellas consideraban tres elementos conceptuales básicos, los que implicaban las siguientes distinciones:

i) Entre empresas de bienes o servicios transables y empresas de bienes o servicios no transables en el mercado internacional. Las primeras compiten en los mercados globales y, por lo tanto, son la fuerza motriz de la economía canadiense; en general, se trata de grandes empresas manufactureras que exportan y de las cuales casi todas las restantes actividades dependen para su prosperidad.

ii) Entre la "creación de riqueza" y la "creación de empleos", siendo la segunda el resultado de la primera. El Consejo considera que habitualmente las políticas industriales se han focalizado en empresas de bienes o servicios no transables internacionalmente a fin de dinamizar la creación de empleos, sin tener en cuenta que son las industrias con producción transable y la riqueza que ellas crean las que determinan cuántos empleos se generan en la economía y si esos empleos son o no bien pagados.

iii) Entre empresas locales y empresas de propiedad nacional. Lo que importa para la prosperidad de una región económica no es quién posee las acciones de una empresa, sino si ésta desarrolla sus actividades de mayor valor agregado (diseño del producto, investigación y desarrollo, toma de decisiones de estrategia empresarial, etc.) en la región. El foco de atención de las políticas industriales deben ser las empresas locales, con independencia de quién tenga la propiedad del capital. ${ }^{5}$

A partir de estos principios, que son sumamente discutibles, el Consejo plantea objetivos y propone instrumentos para revitalizar la economía provincial. Los objetivos implican:

i) Reestructurar la industria para incrementar las

\footnotetext{
${ }^{5}$ Un planteamiento similar se puede encontrar en la obra del Secretario del Trabajo de la Administración del Presidente Clinton, Robert Reich. VEase Reich (1991).
}

actividades manufactureras de alto valor agregado, lo que se considera de especial importancia para una economía muy dependiente de actividades que hacen uso intensivo de recursos naturales poco procesados.

ii) Desarrollar o crear empresas manufactureras globales capaces de tener un alcance transnacional, especialmente en actividades industriales con alto valor agregado.

iii) Reorientar la política industrial, eliminando el sesgo que favorece la producción de bienes no transables por pequeñas empresas localizadas en subregiones de lento crecimiento, sin tener en cuenta plenamente la distinción entre actividades creadoras de riqueza y actividades creadoras de empleos.

iv) Invertir en industrias nacientes y de rápido crecimiento, que son fundamentalmente las que hacen uso intensivo de tecnología.

v) Apoyar a empresas que puedan transformarse a mediano plazo en líderes a nivel mundial ( $t$ hreshold companies), ${ }^{6}$ en la producción de bienes y la prestación de servicios transables en el mercado internacional. A este conjunto, necesariamente pequeño, de empresas debe prestar atención fundamental la política que busque fomentar la creación de riqueza; esto implica, en particular, redefinir el papel de los servicios en el desarrollo provincial e impulsar aquellos que tengan la posibilidad de transformarse en actividades transables globalmente.

vi) Impulsar el desarrollo de una cultura propiamente empresarial que favorezca la asunción de riesgos e impulse la creación de nuevas empresas de bienes y servicios transables en el mercado internacional.

vii) Invertir en desarrollo tecnológico y en recursos humanos.

viii) Crear un consenso nacional que permita reducir la renuencia de los empresarios y de los funcionarios gubernamentales a actuar de consuno en la elaboración e implementación de políticas.

A partir de este conjunto de objetivos y líneas estratégicas, el Consejo propuso un conjunto de instrumentos para concretarlos, entre los que destacan:

i) Los incentivos fiscales a la inversión en empresas exportadoras con potencialidades para trans-

\footnotetext{
${ }^{6}$ El programa de Ontario define como threshold companies a cmpresas que tienen la potencialidad de realizar el esfuerzo necesario para transformarse en líderes mundiales. En esa provincia, existirían empresas de este tipo en sectores considerados estratégicos, como los de partes para automóviles, componentes para la industria aeroespacial, circuitos integrados y comunicación electrónica de datos. (Véase Ontario, Premier's Council, 1988).
} 
formarse en líderes mundiales en sus sectores, a la inversión de los trabajadores en empresas eficientes y rentables, a las inversiones adicionales en investigación y desarrollo superiores a la media histórica de las empresas, y a la inversión en acciones de empresas de bienes y servicios transables internacionalmente que realizan su primera emisión de acciones.

ii) El establecimiento de un sistema de compras estatales guiado por una visión estratégica del desarrollo de la provincia.

iii) El impulso a la creación y expansión de fondos financieros para desarrollar productos o mercados de exportación, así como para fomentar la formación de fondos de capital de riesgo que apoyen las etapas iniciales de inversión.

iv) Los subsidios a la contratación de técnicos, científicos e ingenieros por empresas de tamaño medio.

v) El impulso a programas de capacitación.

Las líneas de política y los instrumentos que propone el informe del Consejo son bastante tradicionales -al menos desde la perspectiva de los países latinoamericanos con más experiencia en política industrial-, pero tienen importantes connotaciones. Por un lado, el hecho de que una región altamente desarrollada y que además operaba con la perspectiva de un acuerdo de libre comercio con Estados Unidos a muy corto plazo, considerara posibles y válidos los enfoques activos de política industrial, sugiere que muy posiblemente América Latina descartó con demasiada premura la necesidad de recurrir a políticas industriales.

Por otro lado, y con una significación más sustantiva, algunas de las ideas básicas del informe del Consejo obligan a reflexionar sobre el tipo de empresas en las que debe focalizarse la política industrial en un contexto de globalización e intensificación de la competencia internacional. En particular, a partir de esa idea cabría cuestionar la práctica tradicional en la región de declarar su apoyo manifiesto a la pequeña industria y dar apoyo en los hechos a las grandes empresas de bienes y servicios no transables. La focalización en empresas con potencialidades para globalizar su comercio y sus inversiones directas en el exterior podría ser una acción eficiente y con más perspectivas de crear riqueza, en el sentido indicado por el Consejo del Primer Ministro de Ontario.

\section{El énfasis en políticas horizontales: CEPAL, $\mathbf{1 9 9 0}$}

A mediados de 1990, la CEPAL propuso una estrategia de desarrollo para América Latina y el Caribe; en ella se planteaba un nuevo enfoque de la relación que debía existir entre crecimiento económico y equidad y se sugerían líneas e instrumentos normativos que podrían facilitar la transformación productiva en la región (CEPAL, 1990). Entre los diversos factores que hicieron necesario y viable plantear una nueva visión de cómo se debería encarar el problema del desarrollo y de la equidad en la región, destacan los siguientes:

i) El notorio fracaso de los países de América Latina en combinar crecimiento y equidad, contrastaba con lo sucedido fuera de la región en varios países que habían experimentado un proceso de desarrollo muy dinámico en las últimas décadas y que habían podido combinar una alta tasa de crecimiento con una distribución del ingreso mucho más equitativa que la de la región. Esta parece haber sido la situación predominante en economías como las de China, España, Hong Kong, Istael, Portugal, la República de Corea y la ex Yugoslavia (Fajnzylber, 1990), si bien en ciertos casos en ese proceso afloraron tendencias al deterioro de la distribución del ingreso.

ii) La constante comparación entre las experiencias de las nuevas economías industrializadas del este de Asia y las de la región latinoamericana mostró que en aquéllas había logros muy superiores en términos de una mayor tasa de ahorro, un patrón de consumo menos orientado a bienes suntuarios, una inserción internacional eficiente y una incorporación dinámica e innovadora de progreso técnico a nivel empresarial. La espectacular experiencia asiática ha sido un factor de innegable importancia en la percepción -predominante en América Latina desde comienzos de los años ochenta- de que el patrón de desarrollo de la posguerra está definitivamente agotado.

iii) Las nuevas aproximaciones analíticas al fenómeno de la relación entre crecimiento y equidad muestran la posibilidad de que exista una relación positiva entre ambos: así, la equidad, más que un resultado del crecimiento, sería un requisito para lograrlo, especialmente en el marco de una revolución tecnológica que acentúa el papel de los recursos humanos en la competitividad. La equidad aparece entonces como un real "factor de produccion" al facilitar la consolidación de sistemas nacionales de innovación integrados, lo que tiene máxima importancia en un mercado mundial en el que la competitividad depende fuertemente de la localización de las empresas en diferentes sistemas nacionales de innovación.

iv) La percepción creciente de que existe un amplio universo de políticas económicas cuyos efectos 
sobre la equidad y el crecimiento no son contrapuestos, sino complementarios: así sucede, por ejemplo, con políticas que impulsan la creación de empleos productivos, la difusión de tecnologías y la capacitación de recursos humanos (CEPAL, 1992).

La propuesta de la CEPAL plantea tanto un objetivo fundamental, como la estrategia que permitiría alcanzarlo y criterios para la selección de las políticas que han de aplicarse. El objetivo es el logro de una infegración eficiente de las economías regionales en la economía mundial en un marco de competitividad auténtica -es decir, basada en mejoras de productividad derivadas de la incorporación de progreso técnico- y no espuria. La CEPAL considera que esta última se dio en muchos países de la región en los años ochenta, cuando el mejoramiento de la capacidad competitiva se debió fundamentalmente a reducciones salariales y a la depredación de los recursos naturales.

Para lograr una competitividad auténtica, la CEPAL propone una estrategia que permitiría resolver tres dilemas que dominaron el debate sobre el tema en la región durante los años ochenta: i) superar la dicotomía entre mercado interno y mercado externo mediante una estrategia de inserción competitiva; ii) avanzar en la articulación productiva como mecanismo para evitar opciones ineficientes que signifiquen apoyar algunos sectores productivos en detrimento de otros, y iii) ir más allá de comprobar intereses contradictorios entre los sectores privado y público, propugnando opciones de negociación y concertación entre ellos.

A partir de esos criterios estratégicos, la propuesta especifica una serie de políticas e instrumentos que serían eficaces para lograr una competitividad auténtica a nivel de inserción competitiva, articulación económica interna y concertación de intereses. En lo que se refiere a la política industrial, en particular, plantea tres criterios que permitirían establecer un orden de prioridad para el uso de distintos tipos de políticas: i) En primera instancia, se sugiere utilizar políticas de estimulación de los mecanismos del mercado, para aumentar su transparencia o reducir los costos asociados a la obtención de información por parte de los agentes; ii) en casos de ineficacia de esos estímulos, se propone el uso de políticas de tipo neutro u horizontal que eviten discriminar entre sectores y subsectores productivos, como las que fomentan el fortalecimiento de la base empresarial, las vinculaciones entre la universidad y la industria, el desarrollo y la difusión de tecnología, y la capaci- tación de los recursos humanos; iii) en casos específicos en los que las políticas horizontales tengan un efecto inferior al deseable desde el punto de vista social, se sugiere la adopción de políticas de intervención directa a nivel sectorial, con un horizonte temporal definido y criterios operativos establecidos con anterioridad para decidir cuándo deberá terminar dicha intervención.

El conjunto de políticas de estimulación de mercados, de alcance horizontal y de intervención selectiva parecería ser lo suficientemente amplio como para encarar situaciones muy diversas y dar continuidad a la visión de largo plazo de la CEPAL que propugna políticas estatales activas, aunque ahora tales políticas deberían orientarse a lograr una eficiente inserción internacional de los países de la región, en lugar de un desarrollo básicamente hacia adentro como en el pasado.

Dentro del conjunto de políticas propuestas por la CEPAL destacan las de alcance horizontal. Muchos de los instrumentos sugeridos en sus publicaciones a partir de 1990 se ubican claramente dentro de ese universo, en especial los que se refieren a la generación de empleos productivos, desarrollo tecnológico y formación de recursos humanos, temas que se encuentran en el centro de las posiciones sustentadas por la institución (CEPAL, 1992). Por otra parte, si bien durante la segunda mitad de los años ochenta parecía que las políticas de intervención directa a nivel sectorial deberían quedar excluidas del conjunto de opciones por considerar, los acontecimientos de los años noventa que se analizan más adelante muestran que en la región existe un espacio para las políticas sectoriales, aunque con algunas características diferentes de las tradicionales.

\section{Un alcance más IImitado para la política in- dustrial: Brasil, 1990}

Brasil tiene una de las experiencias de política industrial más largas e importantes en América Latina. Del conjunto de esa experiencia, en este apartado se resaltará sólo lo que se refiere a cambios en el alcance de la política.

En el pasado, con frecuencia se buscó respuesta a problemas de implementación de la política industrial en el aumento del alcance de sus planes, programas e instrumentos. Así, por ejemplo, Brasil pasó de medidas de política que tenían un impacto básicamente sectorial en los años cincuenta y sesenta, a programas más amplios en los años setenta, y formuló políticas 
para complejos industriales completos a mediados de los ochenta. La idea fundamental era que se consideraba ineficiente establecer metas e implementar mecanismos de política que se enfocaran a ramas industriales aisladas, dado que existían importantes vínculos entre muchas de ellas que compartían una cadena productiva o tenían una base tecnológica común. Esos vínculos habían resultado de la densidad de la estructura industrial a la que había llegado Brasil, por ejemplo, en el complejo electrónico constituido por la microelectrónica, la informática, las telecomunicaciones y la electrónica de consumo.

Así, la política industrial establecida en 1988 en ese país tuvo por principal mecanismo los programas sectoriales integrados que abarcaban cadenas productivas completas, e incluían hasta los sectores proveedores de tecnología (Marcovitch, 1990). En este contexto, los instrumentos normativos tendrían que actuar no sólo sobre las actividades principales de un sector industrial, sino también sobre sus proveedores de materias primas, componentes, bienes de capital y servicios productivos. En última instancia, los programas sectoriales integrados buscaban incidir en todas las actividades que determinaban la competitividad de un sector.

Naturalmente, una política de alcance tan amplio era sumamente difícil de implementar, particularmente en condiciones de extrema inestabilidad macroeconómica y de cambio de gobierno. Así, en junio de 1990 se introdujo una modificación importante al emitirse las Directrices Generales de Política Industrial y Comercio Exterior (Brasil, Ministerio de Economía, Hacienda y Planificación, 1990), las que concebían una nueva relación entre agentes productivos y entre mercados, y aminoraba el énfasis que la política industrial brasileña tradicionalmente había puesto en la acción del Estado y el mercado interno.

Con miras a elevar la eficiencia de las actividades de producción y comercialización, las Directrices Generales anunciaban programas para reducir la protección comercial; aumentar el financiamiento al comercio exterior, a la capacitación tecnológica y a la inversión en capital fijo; impulsar la modernización a nivel de las empresas, fortalecer los sectores de vanguardia, y reestructurar los sectores potencialmente competitivos, pero con problemas. Complementariamente, preveían también nuevos instrumentos legales para fortalecer la competencia y la defensa de los consumidores.

El impulso a la modernización de las empresas lo brindaría el Programa Brasileño de Calidad y Productividad ( $\mathrm{PBQP}$ ) que promovía la introducción de métodos de gestión modernos, la capacitación de los recursos humanos, el desarrollo de la infraestructura tecnológica, una mejor articulación institucional y métodos eficientes para controlar y promover mejoras de calidad a nivel productivo y comercial (ONUDI, 1992). Por su parte, las actividades de reestructuración industrial propiamente dichas tendrían el apoyo de programas de competitividad industrial (PCI), que se irían aprobando gradualmente mediante procesos de concertación con el sector privado que se realizarían en grupos ejecutivos de la política sectorial (GEPS).

Las Directrices Generales eliminaron (por ser excesivamente complejos) los programas sectoriales integrados derivados de la política de 1988 y redujeron el alcance que debía tener la política industrial, al focalizarla básicamente en segmentos de la cadena de valor (Porter, 1990). Este enfoque por segmentos significó un cambio importante, ya que revirtió una tendencia de mucho tiempo a aumentar el alcance de las políticas y, con ello, las dificultades asociadas a su aplicación. Aunque el enfoque por segmentos no es necesariamente el más eficiente en todos los casos, permite concentrar esfuerzos y establecer criterios operativos para decidir la conveniencia de continuar o terminar una acción de política.

\section{La crítica a la visión predominante: Japón, 1991}

Durante los años ochenta se fue consolidando una visión del proceso económico y de las estrategias para alcanzar el desarrollo que tendía a dejar de lado la política industrial, ya que se consideraba que los mecanismos de mercado por sí solos podían asegurar el vector de producción más eficiente. Esta visión, que terminó por predominar en las esferas decisorias de la región, se resumía en el Consenso de Washington, en el que habían concordado las principales instituciones financieras internacionales, la administración republicana de los Estados Unidos e importantes líderes de opinión y centros de investigación (Williamson, 1990).

En ese contexto, a fines de 1991 se comenzó a difundir un documento preparado por el Overseas Economic Cooperation Fund (OECF, 1991) de Japón, en el que se critica algunos de los aspectos más importantes de esa visión. Si bien ese documento no hace propuestas de política industrial en un sentido estricto, sus consideraciones sobre cre- 
cimiento y política de crecimiento son de la máxima importancia para los objetivos de este artículo. En esencia, el planteamiento japonés se centra en cuatro puntos:

i) Respecto de las políticas para lograr un crecimiento sostenido, considera que mejorar el clima de inversión a través de la desregulación de actividades económicas tal vez no baste para expandir la inversión y que por lo tanto puede ser necesario acudir a medidas fiscales y monetarias dirigidas directamente a promoverla.

ii) Es necesario lograr un equilibrio entre la liberalización del comercio y el desarrollo industrial. Este equilibrio, cuya importancia no se reconoce en el enfoque convencional, no se puede conseguir automáticamente, pues si bien la liberalización refuerza las ventajas comparativas estáticas, es demasiado optimista suponer que las nuevas industrias (basadas en ventajas comparativas dinámicas) surgirán por el solo accionar del sector privado.

iii) Existen límites muy claros a la eficiencia de los mecanismos con los que operan los mercados financieros. Ello obliga a reconsiderar la posibilidad de aplicar tasas de interés inferiores a las de mercado en casos de inversiones que implican alto riesgo, generan externalidades, originan beneficios sociales, se dan en mercados con información imperfecta o se producen en industrias nacientes.

iv) La privatización no es siempre la solución a los problemas de eficiencia del sector público.

Este conjunto de proposiciones, que contradice en gran medida la visión predominante, resume, en última instancia, elementos de la experiencia de desarrollo de Japón y de las economías recientemente industrializadas del este de Asia, cuyo crecimiento se basó en la cooperación entre el sector privado y un Estado sumamente intervencionista y con elevada capacidad de dirección. Si bien tal capacidad no está presente en la mayoría de los países de América Latina, y la cooperación no ha sido una característica destacada en ella en el largo plazo, la crítica del oECF a la visión predominante sugiere la necesidad de volver a considerar opciones que posiblemente se desecharon con demasiada premura en la región.

\section{La reapariclón de la política sectorial: México, 1992}

La política industrial y de comercio exterior establecida en México a partir de 1989 no se basaba en distinciones entre sectores o ramas industriales. Por el contrario hacía hincapié en medidas normativas de alcance horizontal que influyeran en el conjunto de la industria e incluso en la totalidad de la economía. ${ }^{7}$ Durante la negociación del acuerdo de libre comercio con Estados Unidos y Canadá, se pudo comprobar algunas de las limitaciones de un enfoque basado sólo en políticas horizontales, dado que las negociaciones tuvieron un alcance fuertemente sectorial y sus resultados establecían condiciones diferenciadas para algunas ramas o clases industriales, como sucedió en las industrias automotriz y de computación.

En este clima de resurgimiento de las consideraciones sectoriales, en mayo de 1992 se dio a conocer el Programa para Promover la Competitividad e Internacionalización de la Industria Textil y de la Confección. ${ }^{8}$ Dado el peso de esa industria en la economía nacional (10\% del PIB manufacturero y $850000 \mathrm{em}-$ pleos) y su potencial de expansión en el contexto de un tratado de libre comercio en América del Norte, el programa se propone contribuir a crear un marco operativo más adecuado para los industriales de la rama y sentar bases administrativas y financieras para elevar su competitividad.

El programa textil y de la confección comprende cuatro grandes áreas: comercio exterior, tecnología, organización industrial y financiamiento. En lo que a comercio exterior se refiere, establece políticas con referencia a normas de etiquetado, ajuste de aranceles aplicables a las importaciones de maquinaria y equipo para evitar que éstos lleguen al productor a precios superiores a los que pagan sus competidores internacionales, mecanismos para evitar prácticas desleales de comercio 9 y promoción de exportaciones. En el rubro tecnológico, identifica un conjunto de problemas que afectan al sector y crea un grupo de trabajo integrado por los sectores público y privado para que presente posibles soluciones.

\footnotetext{
${ }^{7}$ Véase, por ejemplo, el capítulo sobre líneas de acción del Programa Nacional de Modernización Industrial y del Comercio Exterior, 1990-1994 (México, Secretaría de Comercio y Fomento Industrial, 1989).

${ }^{8}$ Al mismo tiempo, se publicó también el Programa para Promover la Competitividad e Internacionalización de las Industrias de la Curtiduría y del Calzado; para los fines del presente trabajo, su contenido presenta características muy similares a las del programa que se ocupa de la industria textil y de la confección. (Véase México, Secretaría de Comercio y Fomento Exterior, 1992a y 1992b.)

${ }^{9}$ En este particular, se establecen mecanismos de operación aduanera y de fiscalización para prevenir la importación ilegal de ropa usada, eliminar la subfacturación, reducir los costos de los trámites aduaneros y facilitar el acceso a la información de comercio exterior
} 
En el área de organización industrial, el programa propone medidas para mejorar la estructura y el comportamiento empresarial en el sector. En especial, hace presente que existe poca coordinación dentro del sector entre sus tres grandes eslabones (elaboración de fibras naturales y sintéticas, fabricación de hilados y tejidos, y confección). Esta escasa coordinación y la excesiva fragmentación del rubro de las confecciones impide aprovechar economías de escala y dificulta una oferta flexible que responda a los cambios en la demanda. El aumento de la comunicación y coordinación entre agentes productivos permitiría poner en marcha sistemas de respuesta rápida que permitiesen obtener una calidad total en cada etapa del proceso, eliminar tiempos improductivos y utilizar la tecnología de manera óptima.

El programa considera también que es importante promover coinversiones y alianzas estratégicas con productores y comercializadores internacionales para poder mejorar la capacidad de respuesta del sector a los retos de la globalización. Asimismo, propone impulsar el acceso al crédito por parte de las empresas del sector, lo que se lograría, entre otros mecanismos, mediante condiciones preferentes para la microempresa y la pequeña y mediana empresa, y la promo- ción de la especialización en el sector por parte de bancos comerciales que operen en el ámbito nacional o regional.

La importancia de este programa, cuyo seguimiento lo hará un comité integrado por representantes de los sectores privado y público, radica, más que en aspectos innovadores, en el hecho de que su propia existencia muestra los límites a los que se enfrentan las políticas horizontales. Más allá de algunas políticas con alcance sumamente amplio (por ejemplo, las de apoyo a la educación primaria), los instrumentos de política tienen en su mayoría un alcance muy diferente según el sector al que se apliquen. Como por la limitación de recursos humanos y financieros no es posible formular políticas para todos los sectores de la estructura industrial, es imprescindible establecer criterios de prioridad para dirigir los esfuerzos a algunos de ellos, considerando variables de uso habitual, como su importancia en el empleo, su potencial exportador o la existencia de riesgos importantes para su supervivencia. Más aún, el programa analizado muestra que se ha operado un cambio radical en el conjunto de instrumentos básicos utilizados por la política, como se refleja en la eliminación de los estímulos fiscales y de los mecanismos abiertos de protección comercial.

\section{III}

\section{Conclusiones: los temas permanentes}

De esta revisión de experiencias y propuestas en materia de política industrial, es posible concluir que existen determinados temas que están permanentemente en el debate sobre el particular. En esta sección se presentan algunos de ellos, de gran importancia para la decisión e implementación de políticas, y se sugieren las maneras de encararlos en los años noventa, algunas de las cuales ya fueron mencionadas en secciones anteriores.

La desaparición de la política industrial de alcance sectorial como elemento relevante en la región parece tener más que ver con el impacto de posturas teóricas y con malas experiencias de los países, que con la solución de los problemas que esa política se proponía enfrentar. Por ello, varios países han tomado medidas que implican políticas horizontales, las que al concretarse necesariamente tienen un alcance sectorial. Así, por ejemplo, la "inserción competitiva" de Brasil, el "pasaje a una segunda fase exportadora" de Chile o el "aprovechar las potencialidades de la zona de libre comercio de América del Norte" de México, llevan a tomar medidas o a elaborar programas con alcance estrictamente sectorial. Esto es aun más patente cuando se intenta defender sectores con problemas de competencia (muchas veces desleal), como, por ejemplo, la industria textil chilena ${ }^{10}$ o la industria de confección mexicana.

El alcance que debe tener la política industrial continúa siendo un tema abierto a la discusión y a diversas experiencias normativas, $y$ es positivo que

\footnotetext{
${ }^{10}$ En diciembre de 1992 el Ministerio de Economía de Chile anunció el Plan de Reconversión para la Industria Textil, que busca superar "su creciente rezago competitivo". Véase Estrategia, 1992.
} 
así sea. Si bien la tendencia hacia programas integrales o dirigidos a complejos extremadamente amplios parece haberse revertido, no hay elementos analíticos que permitan determinar a priori cuál es el alcance que resultará más eficiente. En el párrafo anterior se señalaron condiciones que han llevado a la permanencia de enfoques sectoriales, mientras que las medidas a nivel de segmento son importantes, en especial cuando se desea influir en la dinámica empresarial.

La capacidad de implementación de las políticas continúa siendo un elemento de gran importancia para definir su alcance óptimo. En la región, si bien se podría aducir que algunas de las experiencias reseñadas adolecían de fallas de concepción, su impacto distó siempre de lo esperado, debido a la poca capacidad de implementación de los Estados. Esta insuficiencia se ha traducido a menudo en la aseveración de que no es posible elegir "sectores ganadores o perdedores". En realidad, más que errores en la selección de los sectores o subsectores que han de apoyarse, lo que ha habido es falta de capacidad para aplicar medidas, incluso dirigidas a sectores que eran claramente ganadores, en la región y fuera de ella. Por cierto que adoptar exclusivamente políticas horizontales o neutras no soluciona el problema de incapacidad de implementación, y muchas veces lo magnifica al tener que llevar adelante acciones en sectores sumamente disímiles. Pese a sus innegables ventajas de simplicidad, las políticas estrictamente horizontales tienden a alcanzar con bastante rapidez límites determinados por la especificidad sectorial de la tecnología, los mercados, las estructuras organizativas de los empresarios e incluso las negociaciones económicas internacionales.

A nivel de instrumentos de política, no los hay nuevos; más bien se han eliminado los incompatibles con la apertura comercial y la disciplina fiscal. Así, los subsidios fiscales, las cuotas de importación y las tasas de interés preferentes están dejando de utilizarse de manera generalizada, aunque subsisten en casi todos los países de la región para actividades proveedoras de importantes externalidades o para ramas industriales que se consideran claves, como la automotriz. Por otra parte, la privatización de empresas estatales ha reducido el papel que puede cumplir el poder de compra del Estado como instrumento de política.

Si bien hay avances en la reducción de los costos de información y transacción, en muchos países de América Latina existe amplio margen para aplicar este tipo de políticas de estímulo al mercado. Por otra parte, es necesario, encarar con más profundidad la reducción de los costos de coordinación. Esta es la base del éxito de distritos industriales que utilizan sistemas locales de innovación, como lo muestran diversas experiencias de desarrollo regional, por ejemplo, en Italia (Bianchi y Bellini, 1991).

Más allá de las dimensiones prácticas del fracaso de muchas políticas industriales, hay un factor teórico que deriva del cuestionamiento de los análisis tradicionales de organización industrial. ${ }^{11}$ A los problemas teóricos que enfrenta todo análisis de base fundamentalmente casuística, se han añadido dos elementos que han aumentado la insatisfacción con esos análisis. Por una parte, la crítica neoschumpeteriana y de los analistas de "mercados disputables" han mostrado que la estructura industrial es endógena al sistema estructura-conducta-desempeño y que, por lo tan10, no puede ser considerada un mero instrumento de política, como estaba implícito en muchos programas sectoriales.

Por otra parte, la creciente apertura de las economías de la región tiende a volver menos relevante un cuerpo analítico que fue básicamente concebido para una economía cerrada (como lo era Estados Unidos en los años cuarenta y cincuenta). En economías abiertas y en proceso de globalización, hay que revisar muchas de las proposiciones tradicionales sobre relaciones existentes entre barreras a la entrada, concentración, diversificación y diferenciación de los productos, por un lado, y el poder del mercado, por el otro. Además, la revaloración del papel de las empresas transnacionales (en sentido positivo) y de las empresas estatales (en sentido negativo) ha hecho que muchas de las aseveraciones sobre las relaciones entre las variables mencionadas y la forma de propiedad del capital pierdan relevancia para la formulación de políticas, aunque es discutible que hayan perdido validez. Esta situación de crisis del modelo

\footnotetext{
${ }^{11}$ Como se señaló en la sección II de este artículo, gran parte de las políticas sectoriales que se elaboraron en la región, se han basado en los análisis tradicionales de organización industrial. El principal cuerpo de teoría relativamente reciente en los estudios de organizacion industrial -la teoría de los juegos- ha suministrado algunos resultados analíticos interesantes, pero no concluyentes y que aún distan de poder traducirse en instrumentos y enfoques nuevos de política (Norman y La Manna eds., 1992). Pese a cllo, la teoría de los juegos, la teoría de la información, el análisis de jerarquías y los enfoques basados en la corrección de las imperfecciones del mercado constituyen el núcleo analítico más importante, del que se puede esperar resultados que lleguen a tener un impacto relativamente similar al que tuvo el modelo de estructura-conducta-desempeño (véase por ejemplo Katz, 1993).
} 
de análisis de organización industrial contrasta con las perspectivas que la "nueva teoría del desarrollo" ha abierto al incorporar, en sus modelos, elementos cruciales para el análisis de la industria y sus determinantes tecnológicas, como el aprendizaje por la acumulación de experiencia, las economías de escala y la presencia generalizada de externalidades. La inclusión de variables de este tipo en un marco de equilibrio general ofrece posibilidades interesantes para articular elementos tradicionales del análisis industrial y teorías de crecimiento económico, con fundamentos microeconómicos sólidos (Grossman y Helpman, 1991).

Finalmente, cabe destacar que las opciones de política industrial que se consideren viables en la región se verán afectadas por dos procesos que se dan principalmente en los países desarrollados, y en 10 que a América Latina se refiere, básicamente en los Estados Unidos. Por un lado, la evolución que tenga el pensamiento sobre el alcance eficiente de la acción estatal determinará en buena medida la permanencia del Consenso de Washington vigente hasta 1992, y ello, naturalmente, incidirá en la gama de políticas que los gobiemos de la región considerarán eficientes y viables. Por otro, el tipo de escenario hacia el que tienda el sistema de comercio internacional (de apertura multilateral, de bloques relativamente abiertos o de bloques cerrados) influirá apreciablemente sobre el tipo de política industrial aceptable. Si bien siempre es posible que se encuentren soluciones de comercio a problemas de comercio, no sería sorpresivo que las respuestas reales a tales problemas se tuvieran que buscar en las estructuras productivas subyacentes, como lo muestra el proceso de negociación de la Iniciativa sobre Impedimentos Estratégicos suscrita por Estados Unidos y Japón. En un escenario tal, las negociaciones y las represalias comerciales incluirían también consideraciones de estructura y política industrial. Después de todo, las soluciones óptimas (firstbest) sólo son superiores a las soluciones subóptimas (second-best) cuando todos los actores se comportan como si vivieran en un mundo óptimo.

\section{Bibliografia}

Balassa, B., G. Bueno, P. P. Kuczynski y M. H. Simonsen (1986): Toward Renewed Economic Growth in Latin America, Washington, D.C., Institute for International Economics.

Bianchi, P. y N. Bellini (1991): Public policies for local networks of innovators, Research Policy, vol. 20, N5, Amsterdam, Elsevier Science Publishers B, V. (North-Holland), octubre.

Brailovsky, V. (1980): Industrial Growth and International Trade in Mexico, México, D.F., Secretaría de Patrimonio y Fomento Industrial, mimeo.

Brasil, Ministerio de Economía, Hacienda y Planificación (1990): Directrizes gerais para a política industrial e de comércio exterior, Brasilia, 26 de junio.

CEPAL (Comisión Económica para América Latina y el Caribe) (1990): Transformación productiva con equidad, Santiago de Chile, Publicación de las Naciones Unidas, $\mathrm{N}^{\circ}$ de venta S.90.11.G.6.

(1992): Equidad y transformación productiva: un enfoque integrado, LC/G.1701, Santiago de Chile.

Chami Batista, J. (1992): Debt and Adjustment Policies in Brazil, Boulder, Cotorado, Westview Press.

Choi, D, W. (1993): From Export-Led to Technology-Oriented Growth: the New Korean Industrial Policy, La Jolla, Californiu, IR/PS, University of California, San Diego.

Dias Carneiro, D. (1990): Crise e esperança: 1974-1980, M. de Paiva Abreu (cd.), A ordem do progresso. Cem años de politica econômica republicana, 1889-1989, Río de Janeiro, Editora Campus.

Estrategia, Santiago de Chile, 1 de diciembre de 1992.

Fajnzylber, F. (1990): Industrialización en América Latina: de la "caja negra" al "casillero vacio", Cuadernos de la CEPAL, $\mathrm{N}^{\circ}$ 60, Santiago de Chile, CEPAL. Publicación de las Naciones Unidas, $\mathrm{N}^{\circ}$ de venta S.89.II.G.5.
Grossman, G. y E. Helpman (1991): Innovation and Growth in the Global Economy, Cambridge, Mass., The MIT Press.

Kaldor, N. (1966); Causes of the Slow Rate of Economic Growth of the United Kingdom: an Inaugural Lecture, Londres, Cambridge University Press.

Katz, J. (1993): Falla del mercado y política tecnológica, Revista de la CEPAL, $\mathrm{N}^{\mathrm{a}}$ 50, LC/G. 1767-P, Santiago de Chile, CEPAL, agosto,

Lim, L. Y. C. (1993): Technology Policy and Export Development: the Case of the Electronics Industry in Singapore and Malaysia, Maastricht, Países Bajos, Universidad de las Naciones Unidas, Institute for New Technologies.

Marcovitch, J., (1990): Política industrial e tecnológica no Brasil: uma avaliaçao preliminar, Pensamiento lberoamericano, $\mathrm{N}^{\circ}$ 17, Madrid, Instituto de Cooperación Iberoamericana (ICI)/ Sociedad Estatal Quinto Centenario, enero-junio.

México, Secretaría de Comercio y Fomento Industrial (1989): Programa Nacional de Modernización Industrial y del Comer. cio Exterior, 1990-1994, México, D.F.

(1992a): Programa para promover la competitividad $e$ internacionalizacion de la industria textil y de la confección, México, D.F.

(1992b), Programa para promover la competitividad $e$ internacionalización de las industrias de la curtiduria y del calzado, México, D.F.

Norman, G. and M. La Manna (eds.) (1992): The New Industrial Economics. Recent Developments in Industrial Organization, Oligopoly and Game Theory, Aldershot, Hants, Reino Unido, Edward Elgar Publishing Limited.

OCDE (Organización de Cooperación y Desarrollo Económicos) (1987): Structural Adjustment and Economic Performance, Paris.

OECF (Overseas Economic Cooperation Fund) (1991); Issues Re- 
lated to the World Bank's Approach to Structural Change. Proposal from a Major Partner, OECF Occasioual Paper, $N^{\circ} 1$, octubre.

Ontario, Premier's Council (1988): Competing in the Global Economy, Report of the Premier's Council, Toronto, Queen's Printer for Ontario.

ONUDI (Organización de las Naciones Unidas para el Desarrollo Industrial) (1992): Brazil's Industrial Policy: an Assessment in the Light of the International Experience, PPD. 26, Viena, Servicio de Estudios Nacionales y Regionales, División de Políticas y Perspectivas Industriales.

Peres, W. (1989): Unt decenio de planeación industrial en México,
1979-1988, La Jolla, California, Center for U.S.-Mexican Studies, University of California, San Diego.

Porter, M. (1990): The Competitive Advantage of Nations, Nueva York, The Free Press.

Reich, R. B. (1991): The Work of Nations. Preparing Ourselves for the 2Ist-Ceniury Capitalism, Nueva York, Vintage Books.

Scherer, F. M. y D. Ross (1990): Industrial Market Structure and Economic Performance, Boston, Houghton Mifflin.

Williamson, J. (1990): What Washington means by policy reform, J. Williamson (ed.), Latin American Adjustment: How Much Has Happened?, Wushington, D.C., Institute for International Economics. 